\section{Endogener zirkadianer Schrittmacher}

Helga Peter ${ }^{1}$ und Thomas Penzel ${ }^{2}$

${ }^{1}$ Marburg, Deutschland

${ }^{2}$ Interdisziplinäres Schlafmedizinisches Zentrum, Charité Universitätsmedizin Berlin, Berlin, Deutschland

\section{Synonyme}

Masterzeitgeber

\section{Englischer Begriff}

endogenous circadian pacemaker

\section{Definition}

Bezeichnung für einen innerorganismischen Mechanismus mit Sitz im suprachiasmatischen Nukleus (SCN), der die periodischen Prozesse kontrolliert, die mit dem $\downarrow$,Zirkadianer Rhythmus" des Menschen zusammenhängen. Um die koordinierende Funktion im Rahmen der sogenannten Inne- ren Uhr bzw. der Inneren Uhren zu charakterisieren, wird er besser auch als Masterzeitgeber bezeichnet. Der SchlafWach-Zyklus mit einer Zykluslänge von ca. 24 Stunden unterliegt dem Einfluss des endogenen zirkadianen Schrittmachers, mit einem Optimum für Schlaf und Erholung bei Nacht und für Aktivität und > „Leistung“ in der Wachphase bei Tageslicht. Ebenso werden eine Reihe weiterer physiologischer Parameter durch ihn entscheidend beeinflusst, wie beispielsweise die Körperkerntemperatur, die Kortisolfreisetzung, die Wahrscheinlichkeit für das Auftreten von REMSchlaf, die Kaliumausscheidung im Urin, Aufmerksamkeit und kognitive wie auch psychomotorische Leistung.

Siehe auch

- „Autonomes Nervensystem“

- $>$ „Chronobiologie“

- „Endokrinium“

- $\triangleright$ "Genetik“

- „Metabolismus“

- „Melatonin und zirkadianer Rhythmus“

- „Mineral- und Volumenregulation“

- „Schlafregulation“

- „Wachheit und Schlaf“

- „Zeitgeber“

- „Zirkadiane Schlaf-Wach-Rhythmusstörungen“ 